\title{
Naso Alveolar Cleft Reconstruction: Does Patients’ Age Play a Factor in Postoperative Complication Rate?
}

\author{
Almasri Mazen \\ Oral Maxillofacial Surgery, Umm Alqura University, Mekka, Saudi Arabia \\ Email: mazen_ajm@yahoo.com
}

Received November 21, 2012; revised December 22, 2012; accepted December 30, 2012

\begin{abstract}
Purpose: The purpose of this report is to compare the outcome of unilateral alveolar cleft reconstruction (ACR) in patients aged $6-8$ years versus older more comprehending patients aged $9-12$ years. Materials and Methods: A chart review (from March 2006 to June 2010) was conducted of patients who had alveolar cleft reconstruction using anterior iliac crest bone graft. All the cases included are non syndromic healthy patients whom had unilateral alveolar cleft and were operated for the first time. A total of 49 healthy patients were included in the study. Twenty-four had their surgery between the age of 6 and 8 (Group A), and twenty-five had theirs between 9 and 12 (Group B). The postoperative recovery and complications were compared between the two groups. Results: In group A, $16 \%(n=4)$ showed minimal wound dehiscence while $8 \%(\mathrm{n}=2)$ had a minor wound infection due to food particles wedged in the wound. In Group B, 20\% $(n=5)$ of the patients presented with minimal wound dehiscence, while $12 \%(n=3)$ had minor wound infection due to food particles wedged in the wound. No significant difference was found between the two groups using Fisher's exact test, two-tailed $p$-value $>0.05$. Conclusion: Age factor was not found to be a factor contributing to ACR complication rate.
\end{abstract}

Keywords: Nasoalveolar Cleft; Graft; Age; Complication

\section{Introduction}

Cleft lip and palate is one of the most common congenital defects. Cleft lip with or without cleft palate has a male predilection, and an incidence of 1:700 live births. There is a significant variation amongst different ethnic groups [1]. Isolated cleft palate has a female predilection and an incidence of 1:2000 live births. Alveolar osseous defects occur in $75 \%$ of the cleft lip and palate cases with variable severity [1,2]. Reconstruction of the alveolar bone defect is of prime importance in restoring lip shape, speech, and esthetics. In addition, restoration of an alveolar osseous condition allows for a favorable eruption of the lateral incisors, canines, or bicuspids, and therefore stable ridge continuity. Autogenous bone graft is considered to be the gold standard for alveolar cleft reconstruction (ACR), and usually takes place between the age of 6 and 12. However, it is still controversial whether it is preferable to perform the ACR surgery at early mixed dentition rather than at late mixed dentition, while the major factor that control the procedure timing is the dental age rather that the chronological [2-4]. Treatment of cleft lip and palate patients is based on multidisciplinary team approach, that include the Oral Maxillofacial Surgery (OMFS) unit, Orthodontics, Pediatric Dentistry, Plastic Surgery, Otorhinolaryngology, Speech therapy,
Audiology, and the coordination team. Cooperation of the OMFS, Orthodontics, and the Pediatric Dentistry unit is essential in achieving proper ACR, dental alignment, optimal occlusion, dental implant therapy, facial growth follow up, and orthognathics assessment and treatment.

\section{Materials and Methods}

A chart review was conducted of unilateral alveolar cleft patients whom were operated at the Montreal Children Hospital from March 2006 to June 2010. Healthy non syndromic patients, with unilateral alveolar cleft that were not operated before were included in the study. A total of 49 patients were included and divided to Group A that included 24 children aged 6 to 8 years and Group B that included 25 patients aged $9-12$ years old.

The surgical protocol involved two intraoperative teams, one of which harvested the bone graft from anterior iliac crest while the other team performed the ACR procedure. All the patients had preoperative prophylactic antibiotics (1st generation Cephalosporins or Clindamycin) one hour before the surgery and postoperative prescription for 7 days. The hospital stay ranged from one to two nights and follow up visits commenced one week after the surgery and continued for 12 weeks before attempting any further dental or orthodontic procedure. 
Postoperative instructions were carefully presented to the parents and the patients before and repeatedly after the procedure, which included oral hygiene instructions, antibiotics intake, avoiding hard crispy food and strictly use clear liquids intake for two days then full liquids for a week and a puree diet for another two weeks. Any postoperative complications were recorded and tracked.

\section{Results}

A comparable results were found as in Group A, 16\% (n $=4)$ showed minimal wound dehiscence while $8 \%(n=2)$ had signs of wound infection on top that were successfully treated by local wound care (irrigation, and Chlorhexidine $0.12 \%$ mouth wash) and systemic antibiotics respectively. The infection was likely due to the wedge of some food particles in the wound that were washed out by thorough irrigation with normal saline. On the other hand, Group B had 20\% $(\mathrm{n}=5)$ minimal wound dehiscence, and $12 \%(n=3)$ had local wound infection likely due to the same reason in Group A, that were treated successfully the same way. No significant difference was found between the two groups using Fisher's exact test, two-tailed $\mathrm{p}$-value $>0.05$.

\section{Discussion}

Cleft lip and palate is one of the deformities that can be presented in variable facial severity and hence is extremely difficult to control confounding factors to compare for a study. In our study, the aim was to identify if the patients' age per se can be a factor that changes outcomes. What makes the challenge even more demanding, that the treatment of the cleft lip and palate requires thorough discussion between the physicians and the family in order to customize a suitable plan to fit the particular needs of each case and the parents beliefs as well $[5,6]$. ACR is usually considered after the development of the maxillary permanent lateral incisor or cuspid. Once the root of a non-dysmorphic tooth reaches $1 / 2$ to $2 / 3$ of its original length, alveolar reconstruction should be attempted. However, if partial eruption of the tooth into the cleft occurs prior to the graft placement, an unfavorable periodontal outcome to the erupting tooth is highly anticipated $[3,4]$.

The surgical goals of naso-alveolar reconstruction are the following: separating the nasal mucosal layer from the oral layer, achieving proper closure of the nasal mucosa, nasal floor reconstruction, alveolar cleft grafting, and tension free closure of the oral layer with or without vestibuloplasties [3]. The objective of doing so is to create alveolar continuity environment for the unerupted tooth to erupt, improve the shape of nasal alar base, optimize the lip support, and improving speech capabilities $[3,4]$.
There has been some controversy about the appropriate timing for performing the ACR procedures. Proponents of late mixed grafting (age 9 - 12) claim better canine management while proponents to early mixed grafting (age $6-8$ ) claim the better anterior teeth orthodontic and periodontic management outcome $[7,8]$, reduction of the overall treatment time $[9,10]$, and that simultaneous osseous and soft tissue distraction can be more easily achieved if maxillary expansion takes place after early alveolar grafting [11].

Murthy and Lehman reported on their larger case series of 70 patients, that the complications encountered were infection $(n=3)$, fistula $(n=3)$, pain $(n=4)$, bone graft exposure that led to failure $(\mathrm{n}=2)$, and patients required reoperation for bone grafting $(\mathrm{n}=2)$.

These authors concluded that the infection rate did not show any correlation to patient age or cleft type [12], however, the study did not spot the light on the possible factors contributing to the postoperative complications. J. Lilja et al. [13] reported that grafting at the age of $7-9$ years old led to complete orthodontic cleft closure in $100 \%$ of those patients compared to $91 \%$ of those patients in the $9-11$ years old group. This article suggests that early mixed dentition alveolar grafting ( $6-8$ years) whose purpose was the eruption of healthy lateral incisors into the grafted sites provides better postsurgical outcome and shorter overall treatment time than delaying grafting to 9 - 12 years old.

As this study is reporting a pilot sample that was originally looking at the postoperative complication rate between two different age groups, the results were comparable, and hence, age was not found to be a factor contributing to postoperative complication in our series.

\section{Acknowledgements}

The authors would like to thank the McGill University Cleft Lip and Palate team, Dr. Stephane Schwartz, Vu, Dr. Kapala, and Dr. Schildkraut, for providing the authors with all the needed information. As well, the author would like to express deep appreciation to Mrs. Maria Coirazza, our cleft team coordinator, for her outstanding help in the inter team communication. Last but not least, deep appreciation to Umm Alqura University for their usual support to research activities.

\section{REFERENCES}

[1] B. J. Costello and R. L. Ruiz, "Comprehensive Treatment Planning and Primary Repair," 2nd Edition, BC Decker, Hamilton, 2004, pp. 839-858.

[2] M. Melnick, D. Bixler, P. Fogh-Anderson, et al., "Cleft Lip Palate: An Overview of the Literature an Analysis of Danish Cases Born between 1941-1968," American Journal of Medical Genetics, Vol. 6, No. 1, 1980, pp. 83-87. doi:10.1002/ajmg.1320060108 
[3] M. W. Ochs, "Alveolar Cleft Bone Grafting (Part II): Secondary Bone Grafting," Journal of Oral and Maxillofacial Surgery, Vol. 54, No. 1, 1996, pp. 83-88. doi:10.1016/S0278-2391(96)90311-0

[4] O. G. Da Silva Filho, S. G. Teles, T. A. Ozawa, et al., "Secondary Bone Graft and Eruption of the Permanent Canine in Patients with Alveolar Clefts: Literature Review and Case Report," Angle Orthodontist, Vol. 70, No. 2, 2000, pp. 174-178.

[5] G. Iannetti, P. Cascone, A. Saltarel, et al., "Le Fort I in Clefts Patients: 20 Years' Experience," Journal of Craniofacial Surgery, Vol. 15, No. 4, 2004, pp. 662-669. doi:10.1097/00001665-200407000-00025

[6] J. J. Janulewicz, B. J. Costello, M. J. Buckly, et al., "The Effect of Le Fort I Osteotomies on Velopharyngeal and Speech Function in Cleft Patients," Journal of Oral and Maxillofacial Surgery, Vol. 62, No. 3, 2004, pp. 308-314. doi:10.1016/j.joms.2003.08.014

[7] D. S. Precious, "Alveolar Bone Grafting at 6 Years of Age," Journal of Oral and Maxillofacial Surgery, Vol. 67, No. 10, 2009, pp. 2045-2053. doi:10.1016/i.joms.2009.04.102

[8] G. Semb and T. Ramstad, "The Influence of Alveolar Bone Grafting on the Orthodontic and Prosthodontic Treatment of Patients with Cleft Lip and Palate," Dental Update, Vol. 26, No. 2, 1999, pp. 60-64.

[9] S. Yilmaz, A. R. Kiliç, A. Keles and E. Efeoğlu, "Recon- struction of an Alveolar Cleft for Orthodontic Tooth Movement," American Journal of Orthodontics \& Dentofacial Orthopedics, Vol. 117, No. 2, 2000, pp. 156-163. doi:10.1016/S0889-5406(00)70226-5

[10] T. Ozawa, S. Omura, E. Fukuyama, Y. Matsui, K. Torikai and K. Fujita, "Factors Influencing Secondary Alveolar Bone Grafting in Cleft Lip and Palate Patients: Prospective Analysis Using CT Image Analyzer," Cleft PalateCraniofacial Journal, Vol. 44, No. 3, 2007, pp. 286-291. doi:10.1597/06-054

[11] O. G. da Silva Filho, E. Boiani, A. de Oliveira Cavassan and M. Santamaria Jr., "Rapid Maxillary Expansion after Secondary Alveolar Bone Grafting in Patients with Alveolar Cleft," Cleft Palate-Craniofacial Journal, Vol. 46, No. 3, 2008, pp. 331-338. doi:10.1597/07-205.1

[12] A. Muthy and J. Lehman, "Secondary Alveolar Bone Grafting: An Outcome Analysis," Canadian Journal of Plastic Surgery, Vol. 14, No. 3, 2006, pp. 172-174.

[13] J. Lilja, A. Kalaaji, H. Friede and A. Elander, "Combined Bone Grafting and Delayed Closure of the Hard Palate in Patients with Unilateral Cleft Lip and Palate: Facilitation of Lateral Incisor Eruption and Evaluation of Indicators for Timing of the Procedure," Cleft Palate-Craniofacial Journal, Vol. 37, No. 1, 2000, pp. 98-105. doi:10.1597/1545-1569(2000)037<0098:CBGADC $>2.3$.C $\underline{\mathrm{O} ; 2}$ 